thus leads to results which are too general to be of physical significance. This does not, of course, invalidate von Neumann's general method of averaging over coarse distributions, but only the special case in which the a priori distribution of these coarse distributions is assumed equi-probable.

An alternative proposal put forward in a series of recent papers by an Italian school ${ }^{7}$ is to average, not over all coarse distributions for a fixed initial state, but over all initial states for a fixed coarse distribution. It is assumed that all initial states are a priori equally probable and hence the averaging methods developed by von Neumann may be used. Once again, this treatment is too general to yield any ergodicity criterion; but, unlike von Neumann's method, it does require that the time evolution operator of the system be unitary.

To obtain ergodicity conditions it would be necessary, in both von Neumann's and the Italians' treatments, to make a less strong assumption regarding the a priori distribution, whether of coarse distributions or of initial states. The Italians themselves ${ }^{8}$ have gone some way towards relaxing this strong assumption by replacing the averaging over all initial states of the entire energy shell by separate averaging within each phase cell: the initial states within any phase cell again are assumed equally probable. This procedure does yield ergodicity conditions, but the conditions are somewhat formal and, although they can be linked with the Hamiltonian of the system, the connexion has not been worked out fully. Furthermore, as with the classical criterion of metrical indecomposability, it would appear difficult to determine whether actual physical systems satisfy the conditions. Other ergodicity conditions, which again have a rather tenuous connexion with the Hamiltonian, have been obtained by Landsberg', who considers a class of averaging processes which includes, as special eases, averaging over coarse distributions and over initial states. The class is defined in such a way that averages are independent of particular phase cells and quantum states, and, as with Khinchin's theorem, a condition on the averaging procedure is required.
Ergodic theory has been attacked 10 on the grounds that it is not of physical significance to consider, as it does, an isolated system. Two points may be made here : first, in the attempt to relate dynamical and thermodynamical properties of a system, ergodic theory provides only a first step; once ergodic behaviour has been established, the system of interest, hitherto considered isolated, must be coupled to a large heat-bath, and only the combined system is then regarded as isolated. The interaction between system and surroundings is vital. Secondly, those who favour introducing irreversibility ${ }^{10}$ by way of external influences at the boundaries of a non-isolated system do not appear to be justified in assuming arbitrarily that these extermal influences are random. Although an arbitrary assumption regarding a priori probabilities appears both in von Neumann's and in the Italians' treatments, this assumption refers to methods of handling the system rather than to the system itself. The assumptions therefore are not 'assumptions of disorder', in the sense in which the term was used in early work on kinetic theory.

The work of van Hove ${ }^{11}$ on master equations does not fell within the province of ergodic theory, since it deals with particular systems and seeks to study in detail their time-development. It seems, however, that a generalization of this approach may well prove to be a most fruitful method of studying the approach to equilibrium of arbitrary systems.

1 Birkhoff, G. D., Proc. U.S. Nat. Acad, Sci., 17, 650 (1931).

'Lewis, R. M., Arch. Rational Mech. Anal., 5, 355 (1960).

Khinchin, A. I., Mathematical Foundations of Statistical Mechanics (Dover Publications Inc., New York, 1949).

4 von Neumann, J., Z. Phys., 57, 30 (1929).

- Farquhar, I. E., and Landsberg, P. T., Proc. Roy. Soc., A239, 134 (1957).

- Bocchieri, P., and Loinger, A., Phys. Rev., 111, 668 (1958).

'Bocchieri P. and Loinger, A., Phys, Rev., 114, 948 (1959); Prosperi, G. M., and Scotti, A., Nuovo cimento, 13, 1007 (1959) ; Albertoni, S., Bocchieri, P., and Loinger, A., J. Math. Phys., 1, 244 (1960). - Prosperi, G. M., and Scotti, A., J. Math. Phys., 1, 218 (1960).

'Landsberg, P. T., Proc. Roy. Soc., A (in the press).

${ }^{20}$ Blatt, J. M., Prog. Theor. Phys., 22, 745 (1959).

11 van Hove, L., Physica, 21, 517 (1955) ; 23, 441 (1957) ; 25, 268 (1959).

\title{
TERPENE CHEMISTRY
}

A SYMPOSIUM on terpene chemistry, organized by the Chemical Society, was held in the Chemistry Department of the Imperial College of Science and Technology on February 23 , and attracted an audience of more than two hundred. The programme consisted of six main lectures.

The first session was opened by the president, Sir Alexander Todd, who gave a warm welcome to the speakers and visitors from abroad. Prof. E. R. H. Jones was chairman of the opening session, in which the first paper on "Terpene Compounds with Tenmembered Rings" was given by Prof. F. Sorm, of Prague. After a brief historical introduction, the chemistry of germacrome, a typical ten-membered cyclic terpene, was reviewed in detail, special reference being made to the trans-annular reactions which are so characteristic of this series. A theoretical treatment of the non-classical interaction of double bonds in this series was also provided. Further examples of structural determinations of this class of terpenes were provided by costunolide, balchanolide and isobalchanolide, cnicin, scabiolide and parthenolide.

Later, Prof. B. C. L. Weedon (Queen Mary College) reviewed his recent work on structure determination and synthesis of carotenoids and their biological precursors. Particularly striking results have been achieved with the aid of nuclear magnetic measure. ments carried out and interpreted by Dr. L. M. Jackman at the Imperial College. Examples included spirilloxanthin, spheroidene and the paprika ketones capsorubin, capsanthin and capsanthone. Physical methods have also been applied successfully in determining the structures of a variety of hydrolycopenes, for example, phytoene, $\zeta$-carotene and neurosporene, believed to be biogenetic intermediates of carotenoids. New methods for building up the carotenoid ring systems were also described as part of the syntheses of canthaxanthin and astacene. 
After lunch, Prof. D. H. R. Barton introduced Prof. O. Jeger (Zurich), who discussed the structures of a series of oxidation products of the diterpenes manool and sclareol. Many of these had potential values in the perfumery industry, and evidence for the structures suggested was based on numerous physical measurements as well as chemical degradations and correlations with other diterpene degradation products ; a notable feature of this symposium was the extensive use which chemists are making of nuclear magnetic resonance measurements in structural problems. Prof. H. C. H. Erdtman (Stockholm) then correlated, so far as was possible, the distribution of terpenes among the various orders of the Coniferae and stressed the taxonomic importance of this group of organic compounds. This extensive survey will have far-reaching effects on biogenetic studies of the terpenes; in the course of the work several new terpenes were discovered and the elucidation of these structures was also described.

In the final session, the two papers presented related to the determination of the structure of the complex terpenoid bitter principle, clerodin. Prof. Johnson, as chairman, introduced Prof. Montesth Robertson, who described the potentialities of the X-ray diffraction method in the elucidation of structures of molecules contrining up to one hundred atoms (other than hydrogen).

Prof. Robertson's colleague, Dr. G. A. Sim, then illustrated the general points by discussing the case of clerodin (as a simple derivative) in detail. This elegant work was only one of several similar topics solved by this method in the Glasgow laboratories. Prof. Barton, in his usual lucid style, then discussed the underlying chemistry of this substance and showed how the structure had been derived on the basis of chemical degradations. His work was also complementary to the nuclear magnetic resonance measurements carried out by Dr. Jackman, who described this aspect of the problem. In the ensuing discussion, Dr. K. H. Overton (Glasgow) outlined his elucidation of the stereochemistry of the bitter principle, columbin.

A. W. JOHNSON

\section{AUSTRALIAN ARID ZONE TECHNICAL CONFERENCE}

$\mathrm{A}$

Arid Zone Technical Conference was held at Warburton, Victoria, during December 5-9. It was attended by more than seventy delegates representing very wide range of organizations and sciences. Several overseas visitors were present as observers, including Dr. P. C. Raheja and Dr. Y. Satyanarayan (Central Arid Zone Research Institute, India), Dr. M. H. Aslan (Desert Range Research Station, Egypt) and Mr. M. U. Khan (Meteorological Service, Pakistan).

The programme was organized into nine major sessions. In Session 1, the physical characteristics of the Australian arid zone were outlined in terms of geology (L. C. Noakes, Bureau of Mineral Resources, Canberra), geomorphology (J. A. Mabbutt, Commonwealth Scientific and Industrial Research Organization (C.S.I.R.O.), Canberra), soils (C. G. Stephens, C.S.I.R.O., Adelaide), vegetation (N. C. W. Beadle, University of New England, Armidale), and climate (H. L. Ashton, Bureau of Meteorology, Melbourne). This material was primarily descriptive in nature and provided a continent-wide background for those scientists not directly active in arid zone problems, or concerned with only one geographical locality in the arid zone.

Sessions 2 and 3 were devoted to the principles and problems of plant and animal production and husbandry, and formed the central basis of the conference. Review papers were presented on plant production (R. O. Slatyer, C.S.I.R.O., Canberra), and utilization (R. M. Moore, C.S.I.R.O., Canberra), and on animal production (W. V. Macfarlane, Australian National University, Canberra), and animal husbandry (G. R. Moule, C.S.I.R.O., Prospect and J. H. Whittem, Animal Industry Branch, Alice Springs) and an additional thirty-four contributed papers covered a wide range of specific subjects within these fields of research. In Session 2 data were presented indicating that the existing communities are relatively inefficient in the conversion of rainfall to plant material, and that this phenomenon is due in part to extreme soil mineral deficiency and the physiological and ecological characteristics of the individual species, as well as to the low and intermittent rainfall regime. Experimental data were also presented on the possibilities of introducing new plant species and the problems associated with their establishment, persistence and natural dissemination. This information, together with a recognition of the desirability of concentrating water run-off for more effective use, led to the general concept of concentrating effort for increased plant production on the most favoured parts of the landscape, rather than attempting a blanket improvement of the whole countryside.

The management of the pasture communities under grazing is of special importance under arid conditions, partly because of the need to maintain reserves of perennial evergreen species for drought reserve and partly because of the delicate plant-climate balance and the ease with which communities can be damaged by overgrazing. Also, efficient utilization is influenced by property development, particularly by spacing of watering points and fencing. It was considered that the most advantageous developments in this field can only be achieved if the requirements and responses of the animal are also considered. In consequence, integrated studies of both plants and animals, with the development of range condition standards and management procedures, was recommended.

The main form of commercial production in arid areas in Australia appears likely to remain some form of animal production and this will be restricted mainly to sheep and cattle. In Session 3, it was emphasized. that there is need for more information about both species, but particularly cattle, and with special reference to adaptation, nutrition and reproduction in the more tropical parts of the zone, where there is at present active development of the cattle industry.

As any widespread change in the level of nutrition in the more arid areas will probably be a slow process, emphasis was placed on the need to breed better adapted and better producing animals, either by selection within the genetic material already in the country or by introducing new genes. Evidence was 